\title{
False Positive Result
}

National Cancer Institute

\section{Source}

National Cancer Institute. False Positive Result. NCI Thesaurus. Code C63147.

Problem associated with the device incorrectly reporting that something has been detected and may mislead the operator to take certain actions. 